\title{
Is chaos becoming conversational?
}

Most accounts of chaotic dynamical systems are based on numerical computations of some kind. But now there is a welcome sign that verbal arguments may make these studies more accessible.

THERE is something waywardly biblical about the ambition to make order out of chaos, but there is nothing wrong with wishing to engage in conversation about the concept of chaos in dynamics even if one happens to be so disadvantaged as not recently to have had access to a Cray II. That thought will be provoked in the minds of many who stumble on a fascinating paper called "Routes to chaotic scattering" in the issue of Physical Review Letters for 28 August $(63,919 ; 1989)$.

Chaos in dynamics is a simple concept now much popularized, often for the wrong reasons: there are circumstances in which the solution of the equations describing real problems in dynamics depend sensitively, sometimes exquisitely so, on the initial conditions. The result is that identical particles starting from virtually indistinguishable places in phase space (the join of position and velocity) will rapidly drift apart from each other. Those who work in the field and the Cray II seem to have been made for each other, for most of what is known about chaos is numerical.

What S. Bleher, E. Ott and C. Grebogi from the University of Maryland have done in their article will encourage those with different inclinations. Their arguments are in the main verbal, and almost analytical.

Their problem, which evidently should have been taken up much earlier, is simply that of the scattering of classical particles (otherwise 'billiard balls') by a potential surface, a region of space in which a particle has extra potential energy and from which it is therefore repelled. That such a system should be capable of chaotic behaviour is easily appreciated: even in the oversimple representation of a scattering centre by a billiard ball, the outcome of a scattering event is too often an inconveniently sensitive function of the impact parameter (the offset of the direction of travel of the particle and the parallel line through the centre of the target), as billiards players know all too well.

For kinds of scattering centres other than rigid hard spheres, the behaviour of impinging particles can be more complicated. For example, in nuclear scattering (still classical), there is supposed to be a region of space in which the potential energy is spherically symmetrical about some point (the nucleus). The potential increases steadily as the scattering centre is approached and will then be a maxi- mum, say $E_{\mathrm{m}}$. Rutherford was the first to calculate the scattering angle as a function of the impact parameter.

In this simple case, chaos does not arise. Indeed, if the energy, $E$, of the impinging particles is greater than $E_{\mathrm{m}}$, it is easy enough to guess what the graph of the scattering angle against the impact parameter will be. When the impact parameter is very large and, alternatively, zero, the scattering angle will be zero: particles whose paths are a long way from the scattering centre will not be deflected, whereas those aimed directly at the centre will also go straight through, but there will be some intermediate impact parameter for which the scattering is a maximum, but the maximum angle must be less than $\pi / 2$. The case when $E$ is less than $E_{\mathrm{m}}$ is more complicated, for particles aimed directly at the scattering centre bounce right back, but chaos does not arise.

But what if the scattering centre is not a simple potential-energy peak, but a group of several of them? This is the problem that Bleher and his colleagues tackle, for simplicity in two dimensions. As a numerical example, they describe what happens when the potential energy is a simple function of the coordinates of the form $x^{2} y^{2} \exp \left[-\left(x^{2}+y^{2}\right)\right]$, which is really a system of four equal peaks in the potentialenergy surface. When $E$ is greater than $E_{\mathrm{m}}$, again there is no difficulty; the scattering angle is a more complicated function of the impact parameter, but still smooth. But if the energy is less than the maximum, the system lapses into chaos. What happens is that particles are trapped within the system, bouncing repeatedly between the energy maxima before eventually escaping in a direction that is exquisitely sensitive to the manner in which they were originally captured.

What can be said in words about such a complicated problem? A great deal, it seems. The authors take the general case of an array of three scattering centres whose energy maxima are $E_{\mathrm{m} 1}, E_{\mathrm{m} 2}$ and $E_{\mathrm{m} 3}$, arranged in order of increasing size. Two cases arise, the authors say, depending on the geometrical relationship of the scattering centres. Either the weakest of the three lies outside the circle whose diameter is the line joining the other two, or it lies inside that circle. The essence of the distinction is that, in the first case, the angle at the apex of the triangle joining the three scattering centres must be less than $\pi / 2$, but otherwise is greater than that.
The problem is to predict when and how particles impinging on this system will behave chaotically.

Chaos plainly cannot arise unless $E$ is less than $E_{\mathrm{m} 2}$, for then there is no way in which the particles can be trapped into orbits bouncing between a pair of scattering centres. And, in this case, because the maximum scattering angle at the lowest peak must be less than $\pi / 2$, a particle scattered from say $E_{\mathrm{m}}$ towards $E_{\mathrm{m} 1}$ cannot be scattered back towards $E_{\mathrm{m} 3}$. But once the energy of the incident particles falls below $E_{\mathrm{m} 1}$, an infinite number of trapped orbits is accessible, simply because larger angles of deflection are immediately possible. There is an abrupt transition from order to chaos.

The second case, where the scattering centre with the smallest energy lies within the circle, is more complicated. If $E$ is greater than $E_{\mathrm{m} 1}$ but less than $E_{\mathrm{m} 2}$, everything hangs on whether $E_{\mathrm{m} 1}$ is large enough to deflect a particle arriving from one of the other scattering centres towards the third. If so, there will again be an immediate transition to chaos, for an infinite number of nearly periodic bouncing orbits will be accessible to particles. If, on the other hand, $E_{\mathrm{m} 1}$ is insufficiently large for that, there will still be trajectories in which a particle first scattered by $E_{\mathrm{m} I}$ will be trapped for less or more time between the other two centres. The number of these orbits will multiply rapidly as $E$ is reduced below $E_{\mathrm{m} 2}$, but in a manner exactly like the appearance of chaos in more familiar chaotic systems, by the process called bifurcation (equivalently, 'period-doubling') in which extra allowable orbits make their appearance as some parameter is varied.

The result is simple enough, but plainly applicable to much more complicated arrays of scatterers. To those working in the field of chaos, much of the interest will lie in the authors' demonstration that chaos can arise in the same dynamical system in two distinct ways - either abruptly, or by the more familiar bifurcation route. For what it is worth, their calculation is that the fractal dimension of the number of chaotic orbits varies as they expect with the difference between $E$ and the energy of the scattering peak. But, to others, it will be enlivening that the authors have been able to make their intricate argument almost verbal. Those without an allocation of time on a Cray II will be pleased.

John Maddox 